\title{
A case of interleukin-6-producing cardiac myxoma resembling multicentric Castleman's disease
}

\author{
Atsutomo Morishima, MD,* Akira Marui, MD, PhD, $*$ Takeshi Shimamoto, MD, Yoshiaki Saji, MD, \\ Takeshi Nishina, MD, PhD, and Masashi Komeda, MD, PhD, Kyoto, Japan
}

Cardiac myxoma sometimes presents constitutive symptoms such as fever and weight loss because of a production of interleukin-6 (IL-6). ${ }^{1,2}$ Castleman's disease (CD) presents constitutional symptoms with lymphadenopathy, plasma cell infiltration, polyclonal hypergammaglobulinemia, and inflammatory reaction, which has been reported to be caused by an overproduction of IL- $6 .{ }^{3-5}$ Here, we report a rare case of IL-6-producing cardiac myxoma resembling multicentric $\mathrm{CD}$ with plasma cell infiltration.

\section{CLINICAL SUMMARY}

A 78-year-old man had weight loss, low-grade fever, and advanced anemia for 6 months. After he was referred to our hospital, gastrointestinal examination revealed no malignant lesions. A chest $\mathrm{x}$-ray film showed no pulmonary congestion or cardiomegaly (cardiothoracic ratio $=48 \%$ ). Findings on an electrocardiogram were not remarkable. Laboratory data showed low white blood cell count, anemia, and a severe inflammatory reaction (c-reactive reaction and erythrocyte sedimentation rate; Figure 1). The laboratory data also revealed polyclonal hypergammaglobulinemia and hypoalbuminemia (Figure 1). We initially suspected myelodysplastic syndrome because of the constitutive symptoms and hematologic abnormalities; however, the chromosome examination revealed no abnormality. On further examination, abnormal plasma cell infiltration, erythroid hypoplasia in bone marrow (Figure 2), and a marked increase in plasma IL-6 $(26.2 \mathrm{pg} / \mathrm{mL}$, normal $\leq 4 \mathrm{pg} / \mathrm{mL})$ were observed, which led to suspicion of multicentric $\mathrm{CD}$ with plasma cell infiltration. Thus, steroid and anti-IL-6 therapy was scheduled. However, chest computed tomography revealed not only mediastinal lymphadenopathy but also a large mass $(40 \times$ $30 \mathrm{~mm}$ ) in the left atrium. Therefore, we suspected IL-6-producing cardiac myxoma. The echocardiography

\footnotetext{
From the Department of Cardiovascular Surgery, Kyoto University Graduate School of Medicine, Kyoto, Japan.

* A. Morishima and A. Marui equally contributed to this report.

Received for publication March 21, 2008; accepted for publication May 4, 2008; available ahead of print Sept 1, 2008.

Address for reprints: Akira Marui, MD, PhD, Department of Cardiovascular Surgery, Kyoto University Graduate School of Medicine, 54 Shogoin-Kawahara, Sakyo,

Kyoto, 606-8507 Japan (E-mail: marui@kuhp.kyoto-u.ac.jp).

J Thorac Cardiovasc Surg 2009;138:499-501

$0022-5223 / \$ 36.00$

Copyright (c) 2009 by The American Association for Thoracic Surgery

doi:10.1016/j.jtcvs.2008.05.048
}

also showed mobile high-echoic mass with a diameter of $40 \mathrm{~mm}$, attached to intra-atrial septum in the left atrium.

The operation was performed under a standard cardiopulmonary bypass with mild hypothermia. Through the rightsided left atriotomy, a tumor was observed in the left atrium. The tumor had a gelatin-like, smooth, and dark violet surface with a diameter of $40 \mathrm{~mm}$. Subsequently, the right atrium was opened, and the tumor was completely resected from the fossa ovalis. The resected lesion was directly closed. Histologic examination showed the tumor to be cardiac myxoma. Postoperative course was uneventful, and preoperative abnormal laboratory data including IL-6 returned to the normal range.

\section{DISCUSSION}

To our knowledge, this is the first report of an IL-6-producing cardiac myxoma resembling multicentric $\mathrm{CD}$ with plasma cell infiltration. In 1956, Castleman and colleagues initially reported hyperplasia of mediastinal lymph node as $\mathrm{CD}^{3} \mathrm{CD}$ has been classified into 3 histologic variants ${ }^{4,5}$ : (1) hyaline vascular variant, which accompanies hyalinized vessels in the center of lymph follicles; (2) plasma cell variant, which presents severe infiltration of plasma cell in the lymph follicles; and (3) mixed variant, which is a combination of the 2 variants. CD is also clinically classified as unicentric and multicentric CD. The former presents a swelling of 1 lymph node without systemic symptoms. The latter presents swelling of multiple lymph nodes and constitutive symptoms such as fever, weight loss, and fatigue. Patients with multicentric $\mathrm{CD}$ show anemia, inflammation, polyclonal hypergammaglobulinemia, plasma cell infiltration, and hypoalbuminemia. ${ }^{5}$

Although the exact incidence is unknown, $\mathrm{CD}$ is increasingly relevant today due to its association with human immunodeficiency virus and human herpes virus- $8 .{ }^{4}$ In addition, the relationship between multicentric $\mathrm{CD}$ and the elevation of IL- 6 has been pointed out. ${ }^{3-5}$ IL- 6 by itself has paracrine, endocrine, and autocrine growth functions. ${ }^{1}$ IL-6 is a pleiotropic cytokine with a variety of biologic activities, including differentiation of B cell, thymocytes, and T cells; activation of macrophages; and stimulation of hepatocytes to produce acute-phase proteins such as C-reactive protein.

Recognition of the contribution of IL-6 overproduction to the development of multicentric CD has led to attempts to 


\section{Complete blood cell count and immunological data}

White blood cell count
Hemoglobin
Hematocrit
Platelet count
C-reactive protein
ESR
Immunoglobulin-A
Immunoglobulin-G
Immunoglobulin-M
Interleukin-6

Protein fractionation

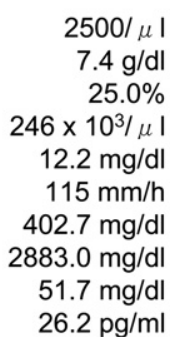

$\downarrow$
$\downarrow \downarrow$
$\downarrow \downarrow$

$\begin{array}{ll}\downarrow & (10.4 \sim 15.9) \\ \downarrow & \downarrow\end{array}$

(1)

$\uparrow \uparrow$
$\uparrow \uparrow$

$\uparrow$

$\uparrow$

$\uparrow \uparrow$

$$
\begin{gathered}
(2900 \sim 8900) \\
(10.4 \sim 15.9) \\
(31.2 \sim 47.0) \\
(100 \sim 345) \\
(\leq 0.2) \\
(\leq 25) \\
(93 \sim 426) \\
(826 \sim 1840) \\
(27 \sim 205) \\
(\leq 4)
\end{gathered}
$$

\section{(1) Albumin \\ (2) $\alpha$ 1-globulin \\ (3) $\alpha$ 2-globulin \\ (4) $\beta$-globulin \\ (5) $\gamma$-globulin \\ A/G ratio}

$\begin{array}{rc}41.1 \% & \downarrow \downarrow \\ 4.2 \% & \uparrow \\ 12.9 \% & \uparrow \\ 10.6 \% & \uparrow \\ 31.2 \% & \uparrow \uparrow \\ 0.7 & \downarrow \\ & \downarrow\end{array}$

(60.2 71.4)

(1.9 3.2)

$(5.8 \sim 9.6)$

(7.0 10.5)

(10.6 20.5)

(1.3 2.5)

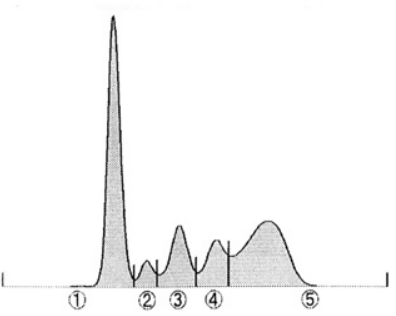

FIGURE 1. Laboratory data. Anemia, severe inflammation, polyclonal hypergammaglobulinemia, and hypoalbuminemia were observed. Interleukin-6 markedly increased. ESR, Erythrocyte sedimentation rate; $A / G$, albumin/globulin; Brackets, normal range.

block IL-6 or its effects directly. The use of a humanized monoclonal antibody to the human IL-6 receptor (tocilizu$\mathrm{mab})^{6}$ has been studied in a series of 28 HIV-negative patients. $^{7}$ Objective improvement was consistently noted in lymphadenopathy and other physical findings, clinical symptoms, and laboratory parameters. Of 15 patients in the series on chronic steroids therapy for CD, $11(73 \%)$ were able to decrease or discontinue its use. Some patients received treatment for up to 3 years, and side effects were mostly mild infusion-related reactions and transient leukopenia. Long-term maintenance with tocilizumab may be beneficial. ${ }^{8}$

The clinical presentation of cardiac myxoma is not specific and depends on their size, location, and mobility. Patients usually present with one or more of the triad of intracardiac obstruction, embolism, and constitutional symptoms. Several studies have shown a relationship between IL-6 plasma level and associated manifestations of cardiac myxoma. Overproduction of IL-6 from cardiac myxoma in- duces constructional symptoms and inflammatory reactions in both adults and children. ${ }^{9}$ The tumor size significantly correlates with the preoperative IL-6 level, and IL-6 levels normalize after the myxoma is excised. ${ }^{1,2,10}$ IL-6 may play an important role in cardiac myxoma recurrence, ${ }^{11}$ whereas "familial myxoma" recurs without immunologic features, inflammatory signs, or elevation of serum IL-6 levels. ${ }^{10}$ The level of IL-6 in the cerebrospinal fluid might be a good marker for the neurologic manifestations of cardiac myxoma. ${ }^{12}$ In addition to IL-6, various cytokines and growth factors, such as vascular endothelial growth factor, basic fibroblast growth factor, and monocyte chemotactic protein-1, were involved in tumor growth and angiogenesis. ${ }^{13}$ Although cardiac myxoma usually presents as a benign neoplasm, these factors sometimes cause it to become malignant (by recurrence, local invasion, and distant metastasis).

As shown in the present report, IL-6 from cardiac myxoma can induce several other conditions such as multiple cerebellar hemorrhages, ${ }^{12}$ pseudovasculitis, ${ }^{14}$ mediastinal
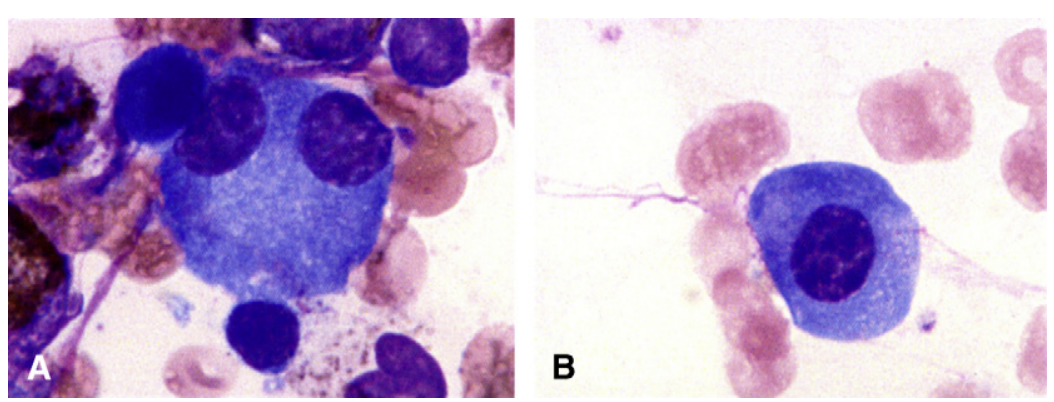

FIGURE 2. Abnormal plasma cell infiltration in bone marrow. A, Binuclear plasma cell. B, Poorly eccentric nuclear plasma cell. Peroxidase staining. 
lymphadenopathy, ${ }^{15}$ and left ventricular hypertrophy. ${ }^{16}$ However, there has been no report of cardiac myxoma with plasma cell infiltration, which suggests $C D$. In the present case, myelodysplastic syndrome was suspected because of constitutional symptoms, low white blood cell count, and severe anemia. However, chromosome examination denied this diagnosis. Plasma cell infiltration and polyclonal hypergammaglobulinemia with elevation of IL- 6 strongly indicated multicentric $\mathrm{CD}$. It was fortunate that the cardiac myxoma was detected just before steroid therapy was begun.

In summary, we experienced a rare case of cardiac myxoma resembling multicentric CD. Cardiac myxoma should be ruled out even if there are poor cardiac symptoms in patients with constitutional symptoms accompanied by hematologic and immunologic abnormalities.

\section{References}

1. Mendoza CE, Rosado MF, Bernal L. The role of interleukin-6 in cases of cardiac myxoma. Clinical features, immunologic abnormalities, and a possible role in recurrence. Tex Heart Inst J. 2001;28:3-7.

2. Keeling IM, Oberwalder P, Anelli-Monti M, Schuchlenz H, Demel U, Tilz GP, et al. Cardiac myxomas: 24 years of experience in 49 patients. Eur J Cardiothorac Surg. 2002;22:971-7.

3. Castleman B, Iverson L, Menendez VP. Localized mediastinal lymph-node hyperplasia resembling thymoma. Cancer. 1956;9:822-30.

4. Dham A, Peterson BA. Castleman disease. Curr Opin Hematol. 2007;14:354-9.
5. Yoshizaki K, Matsuda T, Nishimoto N, et al. Pathogenic significance of interleukin-6 (IL-6/BSF-2) in Castleman's disease. Blood. 1989;74:1360-7.

6. Ohsugi Y. Recent advances in immunopathophysiology of interleukin-6: an innovative therapeutic drug, tocilizumab (recombinant humanized anti-human interleukin-6 receptor antibody), unveils the mysterious etiology of immunemediated inflammatory diseases. Biol Pharm Bull. 2007;30:2001-6.

7. Nishimoto N, Kanakura Y, Aozasa K, et al. Humanized antiinterleukin-6 receptor antibody treatment of multicentric Castleman disease. Blood. 2005;106:2627-32.

8. Akahane D, Kimura Y, Sumi M, et al. Effectiveness of long term administration of humanized antiinterleukin-6 receptor antibody (tocilizumab) for multicentric Castleman's disease with pulmonary involvement. Rinsho Ketsueki. 2006;47: 748-52.

9. Shiraishi I, Yamagishi M, Kato R, et al. A case in a child of giant left-atrial myxoma associated with recurrent high fever and myxoma cells expressing interleukin-6. Eur J Pediatr. 2006;165:346-7.

10. Yokomuro H, Yoshihara K, Watanabe Y, Shiono N, Koyama N, Takanashi Y The variations in the immunologic features and interleukin- 6 levels for the surgical treatment of cardiac myxomas. Surg Today. 2007;37:750-3.

11. Mendoza CE, Rosado MF, Pacheco P. Interleukin-6 production and recurrent cardiac myxoma. J Thorac Cardiovasc Surg. 2001;121:395-6.

12. Yaguchi H, Murakami Y, Sengoku R, Sato H, Inoue K. A case of cardiac myxoma presenting with multiple cerebellar hemorrhages and elevation of interleukin-6 in the cerebrospinal fluid. Rinsho Shinkeigaku. 2004;44:677-81 [in Japanese].

13. Amano J, Kono T, Wada Y, et al. Cardiac myxoma: its origin and tumor characteristics. Ann Thorac Cardiovasc Surg. 2003;9:215-21.

14. Nishio Y, Ito Y, Iguchi Y, Sato H. MPO-ANCA-associated pseudovasculitis in cardiac myxoma. Eur J Neurol. 2005;12:619-20.

15. Takizawa T, Sumino H, Kanda T, Kobayashi I, Nagai R, Ichikawa S. An interleukin-6-producing cardiac myxoma associated with mediastinal lymphadenopathy. Cardiology. 1999;92:275-7.

16. Kanda T, Takahashi T. Interleukin-6 and cardiovascular diseases. Jpn Heart J. 2004;45:183-93 